\title{
Stellar population gradient in lenticular galaxies: NGC 1023, NGC 3115 and NGC 4203
}

\author{
M. Bassin ${ }^{1}$ and Ch. Bonatto ${ }^{1}$ \\ Universidade Federal do Rio Grande do Sul, Instituto de Física, CP 15051, Porto Alegre CEP 91501-970, RS, Brazil \\ Received 11 March 2003 / Accepted 2 July 2003

\begin{abstract}
We investigate the stellar population content in the lenticular galaxies NGC 1023, NGC 3115 and NGC 4203 applying a population synthesis method based on a seven component spectral basis with different ages $-2.5 \times 10^{6}, 10 \times 10^{6}, 25 \times$ $10^{6}, 75 \times 10^{6}, 200 \times 10^{6}, 1.2 \times 10^{9}$ and older than $10^{10}$ years, and metallicity in the range $-1.3 \leq\left[Z / Z_{\odot}\right] \leq-0.2$. This study employs two-dimensional STIS spectra in the range $\lambda \lambda 2900-5700 \AA$, obtained from the Hubble Space Telescope public archives. We extracted one-dimensional spectra in adjacent windows $100 \mathrm{pc}$ wide (projected distance) from the nuclear regions up to $300-400 \mathrm{pc}$. The largest contribution, both in $\lambda 5870 \AA$ flux and mass fraction, comes from old stars (age $>10^{10}$ years). We verified the possible existence of circumnuclear bursts (CNBs) in NGC 3115 and NGC 4203.
\end{abstract}

Key words. galaxies: elliptical and lenticular, cD - galaxies: stellar content - galaxies: nuclei

\section{Introduction}

A precise determination of properties of the stellar content of galaxies is clearly an important issue, since they are related to the galaxy formation and evolution. In order to do this, it is necessary to know what types of stars populate galaxies of different morphological classifications, how the stars are distributed throughout the observable regions of a given galaxy, and how those populations evolve with time. Thus we will be able to explain, for example, why spiral galaxies remain active in star formation throughout their evolution, contrary to what occurs in ellipticals. Consequently, the determination of the stellar ages is a fundamental task.

Despite the recent technological advances associated to observational astronomy, direct observation of individual stars is not yet possible for most of the galaxies, except for the near ones. Consequently, stellar population analysis is based mostly on integrated properties.

The integrated luminosity of a stellar population characterized by a single age and metallicity obviously corresponds to a combination of light emitted by stars with different effective temperatures and surface gravities. A typical galaxy contains a combination of populations defined by a larger range of individual properties with respect to a single population. Therefore, unless we have detailed information on individual stars, significant conclusions on a stellar population can be derived only if the integrated properties are properly modelled. For this reason, population synthesis methods have been developed with

Send offprint requests to: $\mathrm{Ch}$. Bonatto, e-mail: charles@if.ufrgs.br the purpose of determining the star formation history in galaxies based on integrated properties.

Two main approaches have been considered: evolutionary synthesis, a technique to study the spectrophotometric evolution of galaxies (Tinsley 1980; Lacey \& Fall 1985; Arimoto \& Yoshii 1986; Guiderdoni \& Rocca-Volmerange 1987; Clarke 1989; Charlot \& Bruzual 1991; Molla et al. 1997; Boissier \& Prantzos 1999), and stellar population synthesis, a method based on spectra of individual stars (van den Bergh 1975; Pickles 1985) or integrated spectra of star clusters (Bica 1988) that searches for the best mixture of stellar populations that reproduces the observed photometric and/or spectroscopic properties of the galaxy.

In this work we use the stellar population synthesis method developed by Bica (1988), which is based on star cluster spectra of well-defined ages and metallicities. These population templates have ages of $\approx 2.5 \times 10^{6}, 10 \times 10^{6}, 25 \times 10^{6}, 75 \times 10^{6}$, $200 \times 10^{6}, 1.2 \times 10^{9}$ and older than $10^{10}$ years, and metallicity in the range $-1.3 \leq\left[Z / Z_{\odot}\right] \leq-0.2$. However, due to the low signal-to-noise $(\mathrm{S} / \mathrm{N})$ ratio and the uncertainties associated to the metallicity of the population template spectra, we decided to combine templates of a given age with different metallicities. In consequence, the present synthesis method characterizes the stellar populations exclusively by age. Based on this method, we intend to date the successive star formation episodes which took place in the central parts of the lenticular galaxies NGC 1023, NGC 3115 and NGC 4203, and study the variation of the stellar content as a function of distance from the nucleus. In addition, we investigate the potential influence of bars on the star formation rate in the central parts of these galaxies. 
Previous studies confirmed the influence of bars in generating circumnuclear bursts (CNBs) of star formation in spiral galaxies (e.g. Bonatto et al. 1998 and references therein). The effect of bars was already pointed out by Pastoriza (1975) on the basis of visible spectra and images. It has also been predicted by theoretical analysis and numerical simulations of the dynamics in the central regions of spiral galaxies (Athanassoula 1992a,b; Martin 1995; Piner et al. 1995; Elmegreen 1996). The observed association of barred spirals and CNBs favours a scenario in which material is driven along the bar towards the center where, subsequently, star formation is triggered.

In the case of grand-design barred spirals, the occurrence of two inner Lindblad resonances (ILR) is a factor favouring the formation of CNBs: matter expelled in the course of stellar evolution from stars forming the bars will be driven to the region located between both ILR, where it will build up a ring; as soon as the gas density in this ring exceeds the local virial density in the region, star formation will proceed and lead to the CNB. The time it will take for gas in the ring to be exhausted by CNB formation is expected to be quite small. On the other hand, the time for replenishment of gas in the ring, from stellar evolution of stars in the bar, is of the order of $1 \mathrm{Gyr}$ (Elmegreen 1996). Therefore, we may expect CNB activity to take place with a recurrence of about $1 \mathrm{Gyr}$.

To answer the question if the above scenario is also true in the context of lenticular galaxies, we proceed by determining the stellar content of this type of objects. This work is a preliminary study in this direction.

In Sect. 2 we present the galaxies studied. The observations and measurements are described in Sect. 3. We present the population synthesis and discuss the results in Sect. 4. The conclusions of this work are given in Sect. 5 .

\section{Selected galaxies}

The three galaxies (NGC 1023, NGC 3115 and NGC 4203) were selected from a survey in the Space Telescope Science Institute (STScI) public library. We selected objects not classified as peculiars and having no, or faint, nuclear activity. Besides, they satisfy the criterion of having been observed with a long-slit spectrograph, making it possible to extract the spectra along different parts on the slit (corresponding to different spatial regions). The global parameters of the galaxies under consideration are given in Table 1.

\subsection{NGC 1023}

NGC 1023 (Fig. 1) is the brightest member of the homonymous group in Perseus, rich in late-type spirals and dwarf irregulars. It has an extremely bright nucleus in the center of a diffuse bar, situated near the minor axis of the projected image (de Vaucouleurs \& de Vaucouleurs 1964). It is also characterized by the presence of a low luminosity condensation, denominated NGC 1023A, near the eastern end of the major axis. This condensation, discovered by Pease (1917), has caught the attention of Arp (1966) who included NGC 1023 among the family of early-type galaxies with "nearby fragments" in the Atlas
Table 1. Global parameters of the galaxies.

\begin{tabular}{lcccc}
\hline \hline Parameter & NGC 1023 & NGC 3115 & NGC 4203 & Source \\
\hline$\alpha(2000)$ & $02^{\mathrm{h}} 40^{\mathrm{m}} 33.0^{\mathrm{s}}$ & $10^{\mathrm{h}} 05^{\mathrm{m}} 13.9^{\mathrm{s}}$ & $12^{\mathrm{h}} 15^{\mathrm{m}} 05.0^{\mathrm{s}}$ & NED \\
$\delta(2000)$ & $+38^{\circ} 54^{\prime} 00^{\prime \prime}$ & $-07^{\circ} 43^{\prime} 00^{\prime \prime}$ & $+33^{\circ} 11^{\prime} 05^{\prime \prime}$ & NED \\
Type & $\mathrm{SB}(\mathrm{rs}) 0^{-}$ & $\mathrm{S}^{-}$ & $\mathrm{SAB} 0^{-}$LINER & LEDA \\
$M_{\mathrm{B}}$ & -20.23 & -19.77 & -19.48 & LEDA \\
$V$ (radial) $\left(\mathrm{km} \mathrm{s}^{-1}\right)$ & $637 \pm 4$ & $720 \pm 5$ & $1086 \pm 4$ & NED \\
$E(B-V)_{\mathrm{G}}$ & 0.061 & 0.047 & 0.012 & NED \\
Distance $(\mathrm{Mpc})$ & 11 & 14.6 & 18 & LEDA \\
PA $_{\text {phot }}(\mathrm{deg})$ & 87 & 43 & 10 & LEDA \\
Inclination (deg) & 76.7 & 81.6 & 55.4 & LEDA \\
Angular size (arcmin) & $8.7 \times 3.0$ & $7.2 \times 2.5$ & $3.4 \times 3.2$ & NED \\
\hline
\end{tabular}

Table notes. NED: the NASA/IPAC Extragalactic Database is operated by the Jet Propulsion Laboratory, California Institute of Technology, under contract with the National Aeronautics and Space Administration; LEDA: Lyon-Meudon Extragalactic Database, supplied by the LEDA team at the CRAL-Observatoire de Lyon (France).

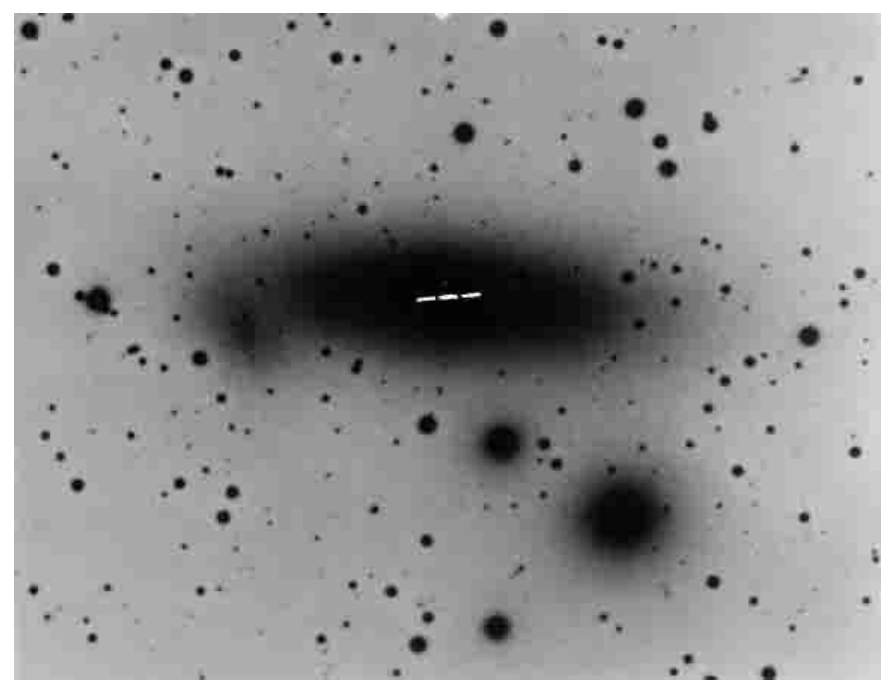

Fig. 1. NGC 1023 and NGC 1023A in a 103aJ plate taken with the 200-inch telescope of Mount Palomar Observatory. The slit position is shown, and the individual spectra were integrated on the interval between the two internal marks on the slit. Scale: $11^{\prime} \times 8.8^{\prime}$. North is at the top and East at left.

of Peculiar Galaxies. Lately NGC 1023A has been interpreted as an individual galaxy, probably a companion of NGC 1023 (Capaccioli et al. 1986)

NGC 1023 gained much interest when it was discovered to be one of the nearest members of the rare class of neutralhydrogen rich lenticular galaxies. It is surrounded by $1.5 \times$ $10^{9} M_{\odot}$ of HI with a rather complex density distribution and kinematics, including two ring-like formations, both showing an overall rotation in the same sense as the stellar body, although in different planes (Sancisi et al. 1984). This material appears not to be associated with regions of star formation, tending to be distributed mainly outside the optical boundaries (van Woerden et al. 1983). The origin of the NGC 1023 neutral hydrogen might be accretion at the expense of intergalactic HI clouds or dwarf companions (Silk \& Norman 1979). Capaccioli et al. (1986) suggest a possible tidal interaction between NGC 1023/1023A that may have removed a large 


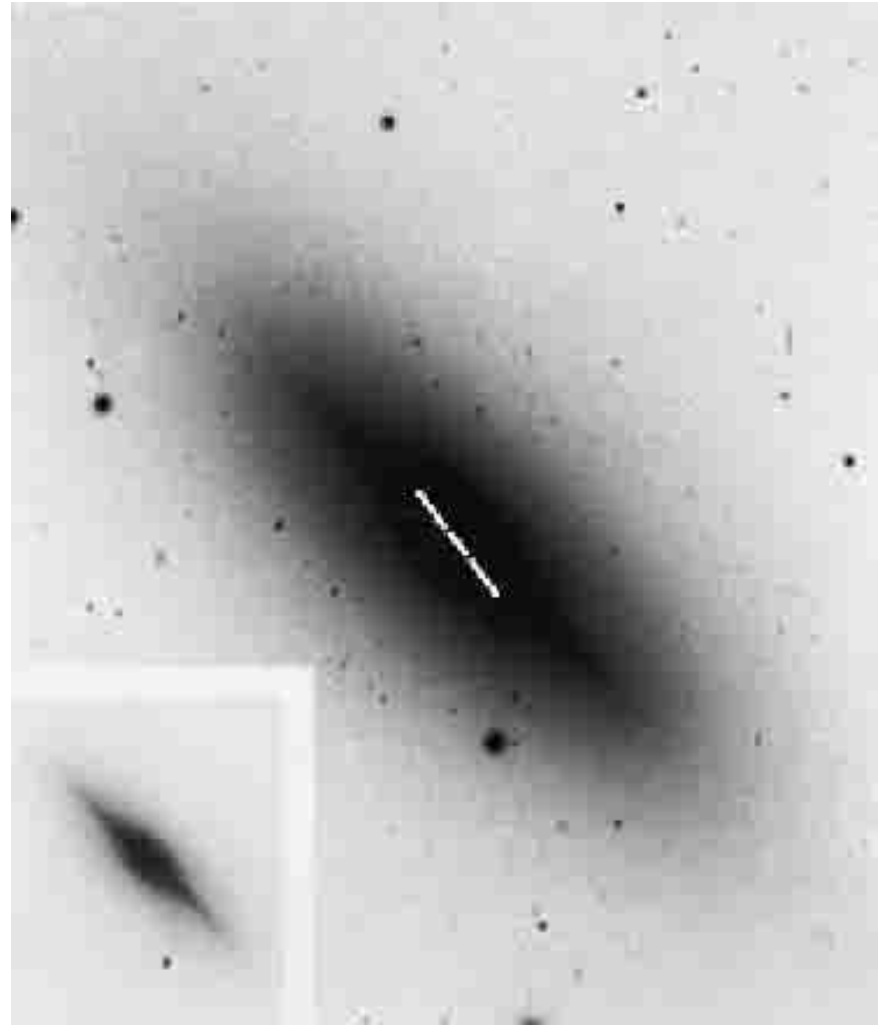

Fig. 2. NGC 3115 in a $103 \mathrm{aO}+\mathrm{WG} 2$ plate taken with the 200 -inch telescope of Mount Palomar Observatory. Same as Fig. 1 for the slit. Scale: $5.8^{\prime} \times 6.8^{\prime}$. North is at the top and East at left.

fraction of the NGC 1023A interstellar medium. There is also the hypothesis that the neutral hydrogen is a remnant of a previous disk mainly consumed by earlier star formation (Larson et al. 1980).

The long-wavelenght spectrum is apparently dominated by $\mathrm{F}$ and G stars. Since NGC 1023 lies at Galactic latitude $b=19^{\circ}$, the strong absorption line of MgII is very likely to be from our Galaxy. In contrast, $\mathrm{MgI}$ is so strong that it is unlikely to be from just the Milky Way interstellar medium, implying the presence of an intrinsic MgI component (Kinney et al. 1993).

Sil'chenko (1999) concluded that the stellar nucleus is substantially younger than the surrounding bulge: the mean age of the nuclear stellar population of NGC 1023 was estimated to be 7 Gyr. The morphological analysis has revealed the existence of a separate circumnuclear stellar disk with a radius of $80 \mathrm{pc}$; probably, the intermediate-age stellar populations are related to these structures.

Modeling of the observed stellar kinematics points to the presence of a central dark compact mass - which presumably is a massive black hole. The models suggest an object with mass $3.9 \times 10^{7} M_{\odot}$ (Bower et al. 2001). Pogge \& Eskridge (1987) detected no $\mathrm{H} \alpha+[\mathrm{N}$ II] emission in the NGC 1023 nucleus.

\subsection{NGC 3115}

NGC 3115 (Fig. 2) is a nearby and well-known galaxy seen almost edge-on, located in the sparse environment of the Leo Spur. It is frequently cited as a transition case between

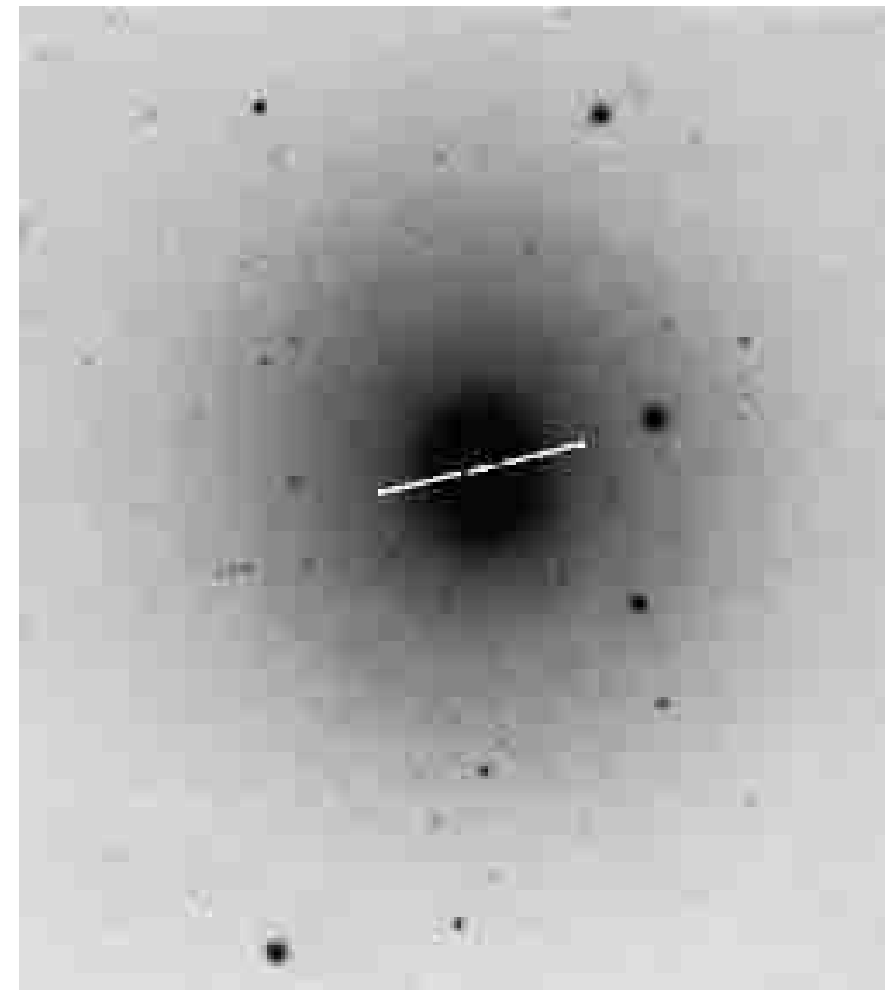

Fig. 3. NGC 4203 in a $103 \mathrm{aO}$ plate taken with the 200 -inch telescope of Mount Palomar Observatory. Same as Fig. 1 for the slit. Scale: $3.6^{\prime} \times 4.1^{\prime}$. North is at the top and East at left.

elliptical and disky galaxies: it has a very prominent bulge and a large, flattened halo; the small optical disk seems to present some evidence reminiscent of spiral arms in the $70^{\prime \prime}-100^{\prime \prime}$ range (Capaccioli et al. 1987).

The disk of NGC 3115 has characteristics similar to those of the spiral galaxies; it has an age intermediate between the disks of typical S0s and those of the typical spirals (Silva et al. 1989). In the bulge, Williams (1976) determined the turnoff at late $\mathrm{G}$ stars, with corresponding age $(9.5 \pm 1.5) \times 10^{9}$ years.

NGC 3115 rotates rapidly about its minor axis, perhaps fast enough to account for its flattened bulge (Illingworth \& Schechter 1982). Investigations found evidence of a supermassive black hole in its center, with an estimated mass of $10^{9} \mathrm{M}_{\odot}$ (Kormendy 2001). Persson et al. (1979) reported the possible existence of an inner ring, on the boundary of the nuclear bulge.

\subsection{NGC 4203}

NGC 4203 (Fig. 3) is a fairly isolated, gas-rich galaxy of normal optical appearance with a high disk-to-bulge luminosity ratio, a small bulge and traces of a bar (de Vaucouleurs \& de Vaucouleurs 1964). Its neutral hydrogen distribution shows an almost circular, inner ring, apparently in circular rotation in the plane of the stellar disk with a radius of $3.8 \mathrm{kpc}$. Outside the optical radius the $\mathrm{HI}$ distribution can be represented by a pseudo-ring, possibly in circular motion in a plane inclined to the optical disk (van Driel et al. 1988). Among the S0 galaxies, inner and outer HI rings are not uncommon but NGC 4203 is rare in having an inner ring without a stellar counterpart. The 
Table 2. Observation log.

\begin{tabular}{lccc}
\hline \hline Galaxy & Date & Exp. time (s) & PA (deg) \\
\hline NGC 1023 & 1997 Nov. 13 & 825 & 92.98 \\
NGC 1023 & 1997 Nov. 13 & 825 & 92.98 \\
NGC 1023 & 1997 Nov. 13 & 825 & 92.98 \\
NGC 3115 & 1998 May 11 & 2373 & 38.42 \\
NGC 3115 & 1998 May 11 & 2373 & 38.42 \\
NGC 4203 & 1999 Apr. 18 & 730 & 105.4 \\
NGC 4203 & 1999 Apr. 18 & 900 & 105.4 \\
\hline
\end{tabular}

preferred scenario for the origin and evolution of the gaseous component is an external origin, i.e. accretion from another galaxy (van Driel et al. 1988).

Classified by Ho et al. (1997) as a LINER 1.9 galaxy, it presents $\mathrm{H} \alpha$ in emission; it is also a faint X-ray nuclear source. The possible existence of a central black hole was suggested, with an estimated mass less than $1.2 \times 10^{7} M_{\odot}$ (Sarzi et al. 2001).

\section{Observations}

The present work uses data obtained from the STScI public archives (eletronic address http: //archive.stsci .edu) which have originally been obtained in other observational programs.

We searched for long-slit spectra observed by the Space Telescope Imaging Spectrograph (STIS). The spectral range covered by STIS is $\lambda 22900-5700 \AA$, using the G430L grism, at the spectral resolution of $\approx 4 \AA$ (dispersion of $2.73 \AA$ pixel $^{-1}$ ). The slit length covers 52 arcsec, the slit width corresponds to 0.2 arcsec and each pixel at the CCD corresponds to 0.051 arcsec in the sky. A log of the observations is given in Table 2.

The primary data reduction - bias and dark subtractions, flat-fielding, wavelength and flux calibrations were previously performed in STScI. We performed additional reduction using standard IRAF $^{1}$ procedures, as follows. Individual twodimensional spectra of a given object were averaged, and cosmic-rays were removed. One-dimensional spectra were extracted in adjacent windows $100 \mathrm{pc}$ wide (projected distance) from the nuclear regions up to $300 \mathrm{pc}$ (in the case of NGC 3115 and NGC 4203) and $400 \mathrm{pc}$ (in the case of NGC 1023) from the nucleus; beyond those distances the low quality of the spectra makes them useless for our purposes. The spectra were corrected for redshift and foreground reddening according to the values given in Table 1.

In order to improve the $(\mathrm{S} / \mathrm{N})$ ratio, we combined the spectra extracted at the same distance on either side of the galactic center. The final $(\mathrm{S} / \mathrm{N})$ ratios at $\lambda 5600 \AA$ ranged between 10 and 20. In Figs. 4 to 6 we present the resulting average spectra of the galaxies, according to extraction region, normalized at $\lambda 5870 \AA$, respectively for NGC 1023 ,

\footnotetext{
${ }^{1}$ IRAF is distributed by the National Optical Observatories, which is operated by the Association of Research in Astronomy, Inc., under cooperative agreement with the National Science Foundation, USA.
}

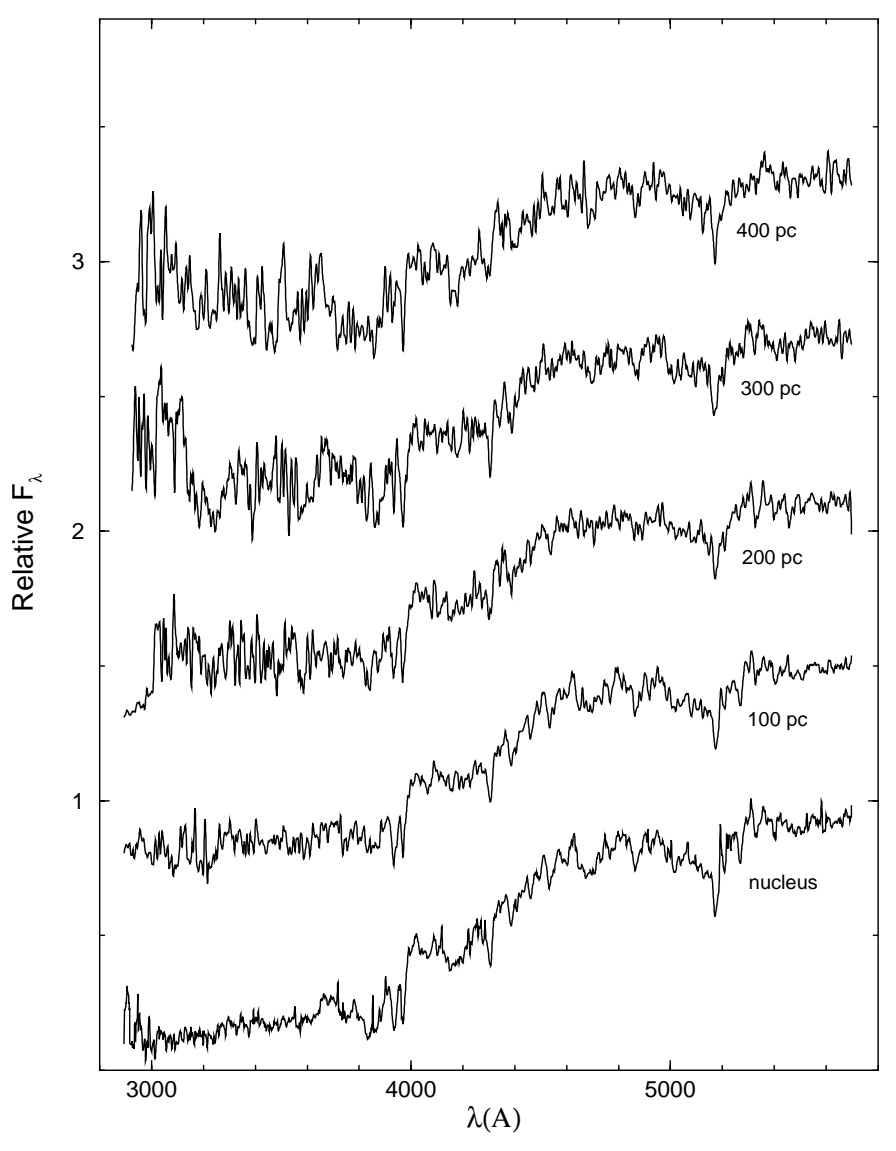

Fig. 4. NGC 1023 spectra. The projected spatial distances from the nucleus are indicated; the distances refer to the center of each extraction. Except for the bottom one, the spectra have been shifted by an arbitrary constant, for clarity purposes.

NGC 3115 and NGC 4203. Notice how the blue to red flux ratio changes from the nucleus to the external regions, particularly in NGC 1023.

\subsection{Measurements}

The age and metallicity of a stellar population are reflected in the features of the observed spectrum. Accordingly, the variation of these spectral properties as a function of distance from the nucleus may give important clues to the spatial distribution of stars in a given galaxy.

In this work we base our stellar population analysis on two kinds of spectral characteristics. First, we use equivalent widths (Ws) of the absorption features Ca IIK $(\lambda \lambda 3908-3952 \AA)$,

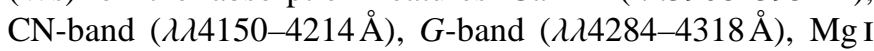
( $\lambda \lambda 5070-5135 \AA), \quad M g H+M g-b a n d ~(\lambda \lambda 5155-5198 \AA), \quad$ Fe I ( $\lambda \lambda 5247-5287 \AA)$ and Fe I $(\lambda \lambda 5314-5354 \AA)$, since these features present significant dependences on metallicity and, particularly, age, which arise from dilution by blue luminous stars in young clusters (Bica \& Alloin 1986a); besides, these features are not disturbed, in general, by emission lines. Second, as additional constraints, we use continuum points (Cs) located at 3220, 3660, 4014 and $5560 \AA$.

In order to measure the Ws for the absorption features, we determined a pseudocontinuum from selected pivot points 


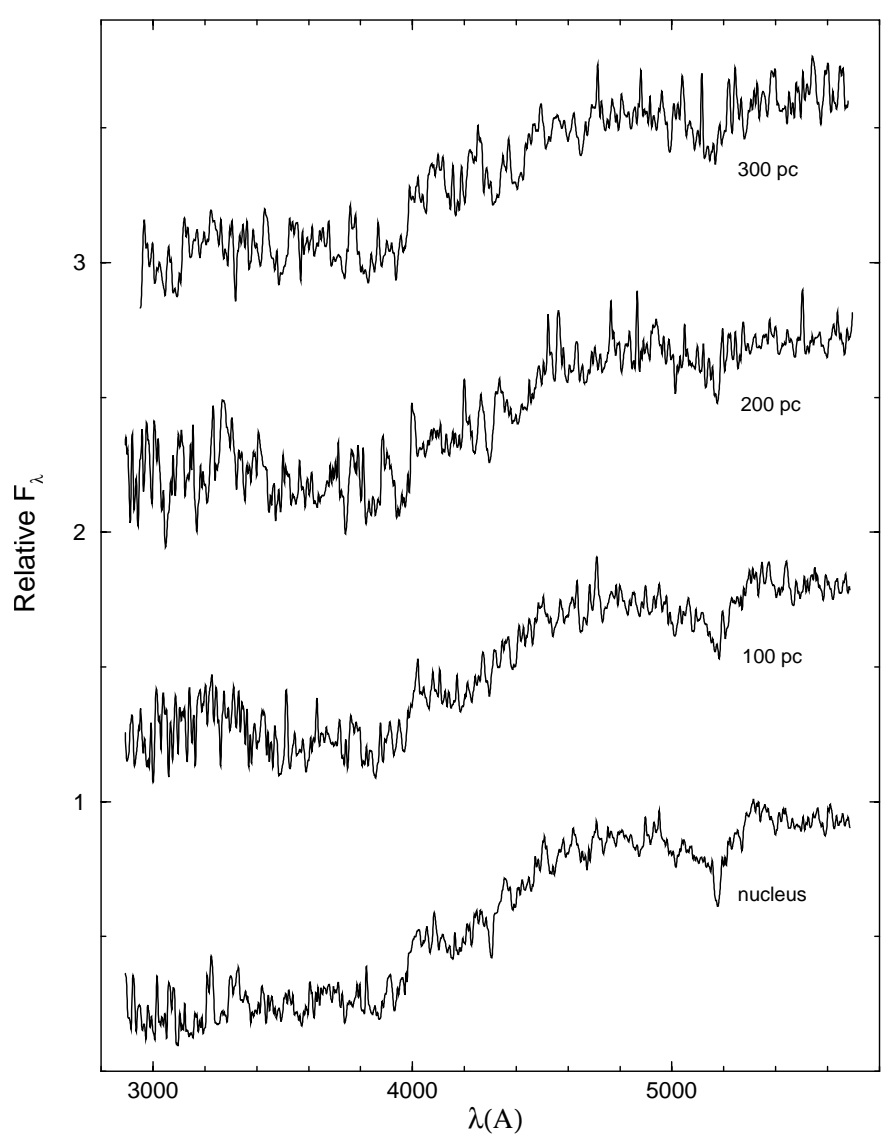

Fig. 5. Same as Fig. 4 for NGC 3115.

around the seven spectral windows described above. The pivot points for the continuum tracing are positioned at 3220,3660 , $4014,4510,5313$ and $5560 \AA$. We measured the Ws by integrating the flux within each spectral window between the traced pseudocontinuum and the spectrum.

Continuum positioning can be somewhat subjective, particularly in the noisier spectra. We evaluate this uncertainty by realizing three independent measurements for each $\mathrm{W}$, allowing for different continuum levels, and then assuming the average value. The observed Ws and Cs along with the corresponding standard deviations are given in Table 3 for each galaxy and extraction. Table 3 also contains the respective synthesized values for the Ws and Cs (see Sect. 4). Ws and continuum definitions are based on Bica \& Alloin (1986a,b), Bica (1988) and Bica et al. (1994).

For NGC 1023 and NGC 3115 the Ws tend to be larger at the nucleus than outside it. For NGC 4203, except for an increase of the Ws for Ca IIK outwards and an anomalous value of the $\mathrm{W}$ for $\mathrm{Mg} \mathrm{I}$ at $200 \mathrm{pc}$, the behaviour of the $\mathrm{Ws}$ is similar.

\section{Population synthesis}

The population synthesis is based on Bica's (1988) algorithm, modified by Schmitt et al. (1996) to include continuum ratios and internal reddening in the search for solutions. In contrast to population synthesis based on stellar libraries, the star cluster method does not require information on the initial mass function and the details of stellar evolution, since these

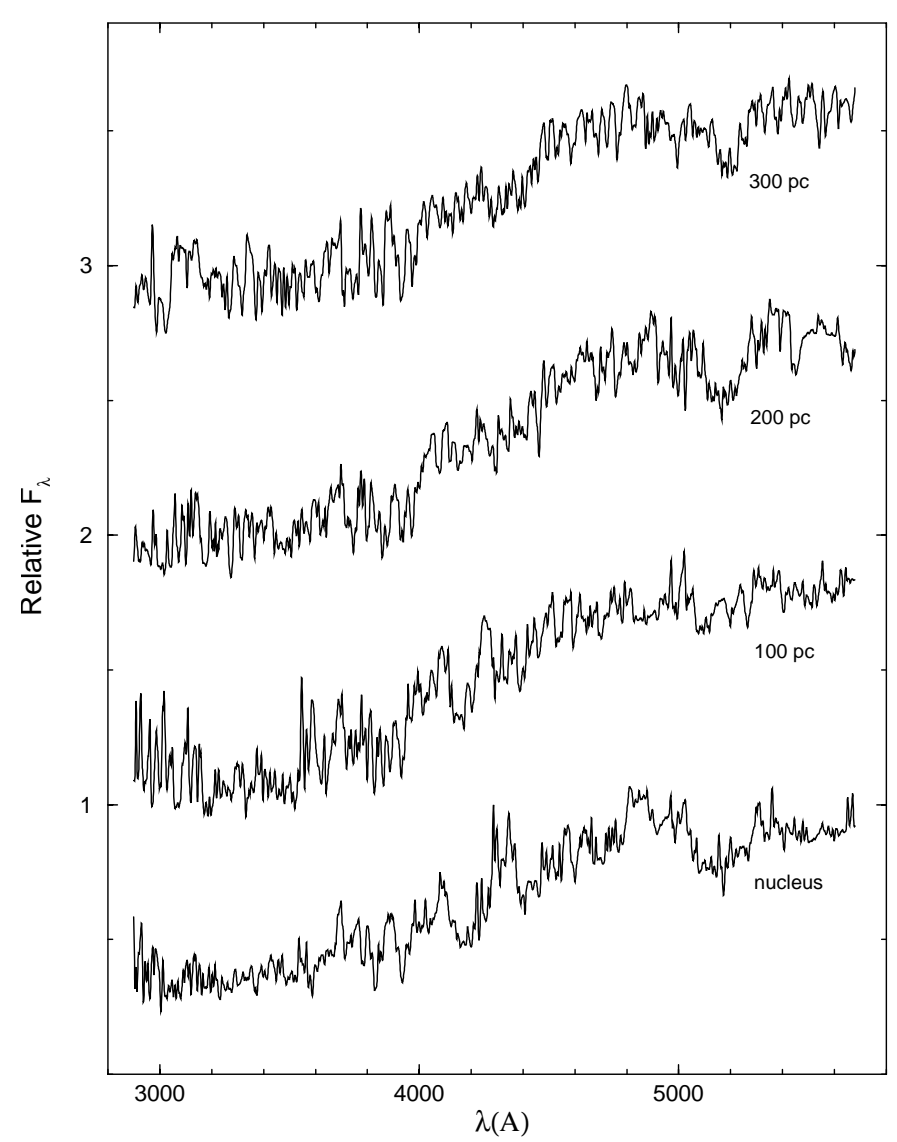

Fig. 6. Same as Fig. 4 for NGC 4203.

assumptions are implicit in real cluster spectra. Consequently, by this approach, the synthesis is reduced to a simpler problem.

Our synthesis includes seven age components, listed in Table 4, and the corresponding spectral templates are presented in Fig. 7. The base elements that we use are taken from the star cluster population templates described in Bonatto et al. (1995).

Basically the algorithm consists of a series of loops, which attributes percentage flux fractions to the base elements at $5870 \AA$, generating all possible linear combinations of these elements according to a given flux contribution step, and subsequently comparing the Ws and Cs of these combinations with the observed values. A combination is an acceptable solution when all the synthesized values (Ws and Cs) reproduce those measured on the galaxy, within allowed windows. These windows are typically of $0.03-0.08$ for $\operatorname{Cs}\left(C_{\lambda} / C_{\lambda 5870}\right)$, which is of the order of the observational errors, and of 1-4 $\AA$ for Ws, or even higher, which reflect both the observational errors and incompleteness of the base elements with respect to the metallicity range. The code also dereddens the observed spectra, testing different values for the internal reddening $E(B-V)_{\mathrm{i}}$ that affects the stellar population.

As a result of the presence of observational errors and the difficulty of building a basis that significantly covers the galaxy features, spectral synthesis is a degenerate problem; in consequence, more than one solution can represent the observed data. The final synthesis solution is thus the average of all acceptable solutions, weighted by their frequency of occurence. 
Table 3. Equivalent width and continuum measurements.

\begin{tabular}{|c|c|c|c|c|c|c|c|c|c|c|c|}
\hline & \multirow[t]{2}{*}{ Extraction: } & \multicolumn{2}{|c|}{ nucleus } & \multicolumn{2}{|c|}{$100 \mathrm{pc}$} & \multicolumn{2}{|c|}{$200 \mathrm{pc}$} & \multicolumn{2}{|c|}{$300 \mathrm{pc}$} & \multicolumn{2}{|c|}{$400 \mathrm{pc}$} \\
\hline & & obs. & synth. & obs. & synth. & obs. & synth. & obs. & synth. & obs. & synth. \\
\hline Galaxy & Window $(\AA)$ & \multicolumn{10}{|c|}{ Equivalent Widths $(\AA)$} \\
\hline \multirow{7}{*}{ NGC 1023} & $3908-3952$ & $17.2 \pm 0.5$ & 17.8 & $14.1 \pm 0.2$ & 15.7 & $11.8 \pm 0.5$ & 13.3 & $11.5 \pm 0.4$ & 13.0 & $10.0 \pm 0.5$ & 11.7 \\
\hline & $4150-4214$ & $13.6 \pm 0.2$ & 8.7 & $11.0 \pm 0.4$ & 7.9 & $10.4 \pm 0.4$ & 6.7 & $9.9 \pm 0.2$ & 6.4 & $8.6 \pm 0.3$ & 6.1 \\
\hline & $4284-4318$ & $8.0 \pm 0.1$ & 8.4 & $8.5 \pm 0.5$ & 7.7 & $7.5 \pm 0.3$ & 7.0 & $7.5 \pm 0.4$ & 6.7 & $6.3 \pm 0.6$ & 6.5 \\
\hline & $5070-5135$ & $11.4 \pm 0.4$ & 8.9 & $11.6 \pm 0.5$ & 8.7 & $8.5 \pm 0.2$ & 7.8 & $7.4 \pm 0.9$ & 7.7 & $9.3 \pm 0.4$ & 7.4 \\
\hline & $5155-5198$ & $11.3 \pm 0.3$ & 10.2 & $10.9 \pm 0.4$ & 9.8 & $9.8 \pm 0.1$ & 9.0 & $9.3 \pm 0.5$ & 8.8 & $10.9 \pm 0.1$ & 8.7 \\
\hline & $5247-5287$ & $4.6 \pm 0.4$ & 4.1 & $4.6 \pm 0.3$ & 3.9 & $1.5 \pm 0.2$ & 3.8 & $2.2 \pm 0.5$ & 3.8 & $3.3 \pm 0.1$ & 3.7 \\
\hline & 5314-5354 & $2.3 \pm 0.5$ & 1.8 & $1.9 \pm 0.4$ & 1.8 & $1.8 \pm 0.2$ & 1.7 & $0.7 \pm 0.3$ & 1.8 & $1.6 \pm 0.2$ & 1.7 \\
\hline \multirow{7}{*}{ NGC 3115} & $3908-3952$ & $17.4 \pm 0.5$ & 16.9 & $12.5 \pm 0.4$ & 13.8 & $13.5 \pm 0.5$ & 13.5 & $12.9 \pm 0.5$ & 13.2 & & \\
\hline & $4150-4214$ & $10.1 \pm 0.3$ & 8.4 & $13.2 \pm 0.2$ & 7.3 & $10.8 \pm 0.7$ & 6.8 & $8.6 \pm 0.5$ & 6.7 & & \\
\hline & 4284-4318 & $8.0 \pm 0.5$ & 8.0 & $4.9 \pm 0.4$ & 7.1 & $8.4 \pm 0.5$ & 7.0 & $7.0 \pm 0.4$ & 6.8 & & \\
\hline & $5070-5135$ & $12.8 \pm 0.4$ & 8.8 & $9.7 \pm 0.5$ & 8.3 & $8.5 \pm 0.8$ & 7.9 & $11.2 \pm 0.3$ & 8.0 & & \\
\hline & $5155-5198$ & $12.2 \pm 0.1$ & 10.0 & $10.4 \pm 0.4$ & 9.5 & $7.3 \pm 0.4$ & 9.0 & $9.0 \pm 0.6$ & 9.1 & & \\
\hline & $5247-5287$ & $4.3 \pm 0.2$ & 4.0 & $3.4 \pm 0.3$ & 3.9 & $2.7 \pm 0.3$ & 3.8 & $4.5 \pm 0.3$ & 3.8 & & \\
\hline & 5314-5354 & $0.8 \pm 0.4$ & 1.7 & $0.6 \pm 0.2$ & 1.7 & $1.7 \pm 0.4$ & 1.7 & $1.5 \pm 0.3$ & 1.7 & & \\
\hline \multirow{8}{*}{ NGC 4203} & $3908-3952$ & $10.7 \pm 0.7$ & 12.0 & $11.3 \pm 0.3$ & 15.4 & $16.7 \pm 0.6$ & 16.1 & $17.0 \pm 0.6$ & 15.4 & & \\
\hline & $4150-4214$ & $15.2 \pm 0.3$ & 7.1 & $15.7 \pm 0.4$ & 7.4 & $8.9 \pm 0.5$ & 8.1 & $7.1 \pm 0.6$ & 7.7 & & \\
\hline & $4284-4318$ & not used & & not used & & $6.5 \pm 0.3$ & 7.8 & $5.7 \pm 0.4$ & 7.6 & & \\
\hline & $5070-5135$ & $12.9 \pm 0.4$ & 8.1 & $11.5 \pm 0.6$ & 8.3 & $15.7 \pm 0.3$ & 7.7 & $10.4 \pm 0.6$ & 8.5 & & \\
\hline & 5155-5198 & $13.0 \pm 0.3$ & 13.7 & $13.0 \pm 0.5$ & 9.5 & $13.3 \pm 0.5$ & 9.9 & $11.9 \pm 0.6$ & 9.7 & & \\
\hline & $5247-5287$ & $5.0 \pm 0.5$ & 3.8 & $4.7 \pm 0.4$ & 3.9 & $4.8 \pm 0.4$ & 4.0 & $4.8 \pm 0.4$ & 3.9 & & \\
\hline & 5314-5354 & $2.1 \pm 0.6$ & 1.6 & $1.0 \pm 0.5$ & 1.7 & $1.8 \pm 0.3$ & 1.8 & $2.9 \pm 0.5$ & 1.7 & & \\
\hline & $\lambda(\AA)$ & \multicolumn{10}{|c|}{ Continuum points $-C_{\lambda} / C_{\lambda 5870}$} \\
\hline \multirow{4}{*}{ NGC 1023} & 3220 & $0.17 \pm 0.01$ & 0.17 & $0.24 \pm 0.01$ & 0.24 & $0.31 \pm 0.02$ & 0.31 & $0.33 \pm 0.02$ & 0.33 & $0.40 \pm 0.03$ & 0.41 \\
\hline & 3660 & $0.27 \pm 0.02$ & 0.27 & $0.32 \pm 0.02$ & 0.31 & $0.35 \pm 0.02$ & 0.36 & $0.36 \pm 0.02$ & 0.37 & $0.45 \pm 0.03$ & 0.43 \\
\hline & 4014 & $0.50 \pm 0.02$ & 0.49 & $0.51 \pm 0.02$ & 0.52 & $0.61 \pm 0.02$ & 0.59 & $0.61 \pm 0.03$ & 0.60 & $0.66 \pm 0.03$ & 0.66 \\
\hline & 5560 & $0.95 \pm 0.02$ & 0.95 & $0.95 \pm 0.02$ & 0.95 & $0.94 \pm 0.03$ & 0.96 & $0.96 \pm 0.03$ & 0.96 & $0.97 \pm 0.03$ & 0.97 \\
\hline \multirow{4}{*}{ NGC 3115} & 3220 & $0.24 \pm 0.02$ & 0.22 & $0.43 \pm 0.02$ & 0.36 & $0.45 \pm 0.03$ & 0.39 & $0.39 \pm 0.03$ & 0.36 & & \\
\hline & 3660 & $0.30 \pm 0.02$ & 0.31 & $0.34 \pm 0.02$ & 0.40 & $0.37 \pm 0.02$ & 0.43 & $0.35 \pm 0.03$ & 0.39 & & \\
\hline & 4014 & $0.52 \pm 0.02$ & 0.52 & $0.53 \pm 0.02$ & 0.58 & $0.58 \pm 0.02$ & 0.61 & $0.67 \pm 0.03$ & 0.63 & & \\
\hline & 5560 & $0.97 \pm 0.02$ & 0.95 & $0.93 \pm 0.02$ & 0.95 & $0.94 \pm 0.03$ & 0.96 & $0.93 \pm 0.03$ & 0.97 & & \\
\hline \multirow{4}{*}{ NGC 4203} & 3220 & $0.37 \pm 0.02$ & 0.40 & $0.24 \pm 0.03$ & 0.24 & $0.25 \pm 0.03$ & 0.24 & $0.25 \pm 0.03$ & 0.26 & & \\
\hline & 3660 & $0.45 \pm 0.02$ & 0.42 & $0.32 \pm 0.02$ & 0.32 & $0.35 \pm 0.03$ & 0.31 & $0.35 \pm 0.03$ & 0.33 & & \\
\hline & 4014 & $0.56 \pm 0.02$ & 0.58 & $0.55 \pm 0.02$ & 0.53 & $0.47 \pm 0.02$ & 0.51 & $0.51 \pm 0.03$ & 0.54 & & \\
\hline & 5560 & $0.94 \pm 0.02$ & 0.96 & $0.93 \pm 0.02$ & 0.95 & $0.91 \pm 0.03$ & 0.95 & $0.94 \pm 0.03$ & 0.93 & & \\
\hline
\end{tabular}

The spectral window 4284-4318 A is contaminated by emission lines ( $\mathrm{H} \gamma$ and [O III]) in the first two extractions of NGC 4203 .

Table 4. Equivalent width and continuum measurements for the base.

\begin{tabular}{|c|c|c|c|c|c|c|c|c|}
\hline & Element/age $\left(10^{6}\right.$ years $)$ : & 2.5 & 10 & 25 & 75 & 200 & 1200 & $>10000$ \\
\hline Window $(\AA)$ & Main absorbers & \multicolumn{7}{|c|}{ Equivalent Widths $(\AA)$} \\
\hline $3908-3952$ & Ca IIK $3933 \AA$ & 0.00 & 1.40 & 1.81 & 1.65 & 5.25 & 8.65 & 18.78 \\
\hline $4150-4214$ & CN-band $4182 \AA$ & 0.00 & 0.84 & 0.94 & 0.72 & 0.81 & 1.32 & 9.12 \\
\hline $4284-4318$ & $G$-band $4301 \AA$ & 0.00 & 0.76 & 1.62 & 2.62 & 3.80 & 5.04 & 8.56 \\
\hline $5070-5135$ & Mg I $5102 \AA$ & 0.00 & 3.20 & 2.42 & 1.20 & 1.32 & 2.91 & 9.11 \\
\hline $5155-5198$ & $\mathrm{MgH}+\mathrm{Mg}$-band $5176 \AA$ & 0.00 & 2.51 & 1.87 & 2.38 & 3.48 & 3.85 & 10.36 \\
\hline $5247-5287$ & Fe I $5270 \AA$ & 0.00 & 2.62 & 1.66 & 2.36 & 2.41 & 2.23 & 4.12 \\
\hline $5314-5354$ & Fe I $5335 \AA$ & 0.00 & 1.33 & 1.21 & 1.96 & 1.89 & 0.75 & 1.81 \\
\hline$\lambda(\AA)$ & & \multicolumn{7}{|c|}{ Continuum points $-C_{\lambda} / C_{\lambda 5870}$} \\
\hline 3220 & & 4.69 & 2.17 & 1.53 & 1.18 & 0.63 & 0.45 & 0.16 \\
\hline 3660 & & 3.27 & 1.37 & 1.07 & 0.88 & 0.63 & 0.48 & 0.28 \\
\hline 4014 & & 2.27 & 1.40 & 1.83 & 1.74 & 1.25 & 0.83 & 0.50 \\
\hline 5560 & & 1.13 & 1.00 & 1.10 & 1.08 & 1.04 & 0.97 & 0.95 \\
\hline
\end{tabular}

Table notes. Column 2: main absorbers in each spectral window. Column 3: the HII region component is represented by a featureless continuum. 
Table 5. Stellar population synthesis results.

\begin{tabular}{|c|c|c|c|c|c|c|c|c|c|}
\hline & Element/a & e (10 years): & 2.5 & 10 & 25 & 75 & 200 & 1200 & $>10000$ \\
\hline Galaxy & Extraction & $E(B-V)_{\mathrm{i}}$ & \multicolumn{7}{|c|}{$\lambda 5870 \AA ̊$ Aflux fraction $(\%)$} \\
\hline \multirow{5}{*}{ NGC 1023} & nucleus & $0.04 \pm 0.02$ & $0.1 \pm 0.3$ & $0.4 \pm 0.6$ & $0.2 \pm 0.4$ & $0.2 \pm 0.4$ & $0.5 \pm 0.7$ & $0.9 \pm 1.0$ & $97.7 \pm 0.8$ \\
\hline & $100 \mathrm{pc}$ & $0.05 \pm 0.02$ & $0.7 \pm 0.7$ & $2.2 \pm 1.6$ & $1.2 \pm 1.2$ & $0.6 \pm 0.8$ & $0.4 \pm 0.6$ & $0.8 \pm 0.8$ & $94.1 \pm 0.9$ \\
\hline & $200 \mathrm{pc}$ & $0.04 \pm 0.02$ & $1.5 \pm 0.5$ & $0.9 \pm 0.9$ & $3.9 \pm 2.0$ & $1.6 \pm 1.7$ & $1.0 \pm 1.1$ & $5.6 \pm 1.2$ & $85.5 \pm 0.8$ \\
\hline & $300 \mathrm{pc}$ & $0.06 \pm 0.03$ & $1.1 \pm 0.8$ & $3.3 \pm 2.3$ & $3.4 \pm 2.6$ & $3.1 \pm 2.4$ & $2.5 \pm 2.3$ & $2.8 \pm 2.4$ & $83.8 \pm 1.8$ \\
\hline & $400 \mathrm{pc}$ & $0.02 \pm 0.01$ & $2.9 \pm 0.6$ & $2.1 \pm 1.7$ & $3.2 \pm 2.3$ & $2.9 \pm 2.3$ & $3.3 \pm 2.6$ & $3.6 \pm 2.9$ & $82.0 \pm 2.0$ \\
\hline \multirow{4}{*}{ NGC 3115} & nucleus & $0.02 \pm 0.01$ & $0.6 \pm 0.6$ & $1.4 \pm 1.3$ & $0.5 \pm 0.7$ & $0.2 \pm 0.4$ & $0.3 \pm 0.5$ & $0.4 \pm 0.7$ & $96.6 \pm 0.8$ \\
\hline & $100 \mathrm{pc}$ & $0.02 \pm 0.02$ & $2.9 \pm 1.5$ & $4.6 \pm 2.6$ & $0.3 \pm 0.4$ & $0.2 \pm 0.4$ & $0.1 \pm 0.2$ & $0.4 \pm 0.6$ & $91.5 \pm 1.1$ \\
\hline & $200 \mathrm{pc}$ & $0.01 \pm 0.01$ & $4.0 \pm 0.0$ & $1.3 \pm 0.4$ & $0.3 \pm 0.4$ & $0.7 \pm 0.9$ & $0.7 \pm 0.9$ & $6.3 \pm 1.3$ & $86.7 \pm 0.5$ \\
\hline & $300 \mathrm{pc}$ & $0.01 \pm 0.01$ & $0.1 \pm 0.3$ & $5.3 \pm 1.1$ & $5.9 \pm 1.7$ & $1.2 \pm 1.4$ & $0.2 \pm 0.3$ & $0.6 \pm 0.7$ & $86.7 \pm 0.6$ \\
\hline \multirow{4}{*}{ NGC 4203} & nucleus & $0.03 \pm 0.02$ & $4.9 \pm 0.3$ & $1.4 \pm 0.9$ & $0.3 \pm 0.5$ & $0.3 \pm 0.5$ & $0.7 \pm 0.8$ & $1.6 \pm 1.5$ & $90.7 \pm 1.3$ \\
\hline & $100 \mathrm{pc}$ & $0.03 \pm 0.01$ & $0.4 \pm 0.5$ & $2.1 \pm 1.3$ & $0.5 \pm 0.6$ & $0.5 \pm 0.8$ & $0.9 \pm 1.0$ & $6.8 \pm 1.3$ & $88.8 \pm 0.4$ \\
\hline & $200 \mathrm{pc}$ & $0.05 \pm 0.02$ & $1.8 \pm 0.6$ & $1.5 \pm 1.6$ & $0.2 \pm 0.4$ & $0.2 \pm 0.4$ & $0.2 \pm 0.3$ & $0.2 \pm 0.4$ & $95.9 \pm 0.8$ \\
\hline & $300 \mathrm{pc}$ & $0.03 \pm 0.02$ & $1.8 \pm 0.4$ & $1.0 \pm 1.2$ & $0.4 \pm 0.6$ & $0.5 \pm 0.6$ & $1.1 \pm 1.0$ & $2.3 \pm 2.1$ & $92.9 \pm 1.7$ \\
\hline
\end{tabular}

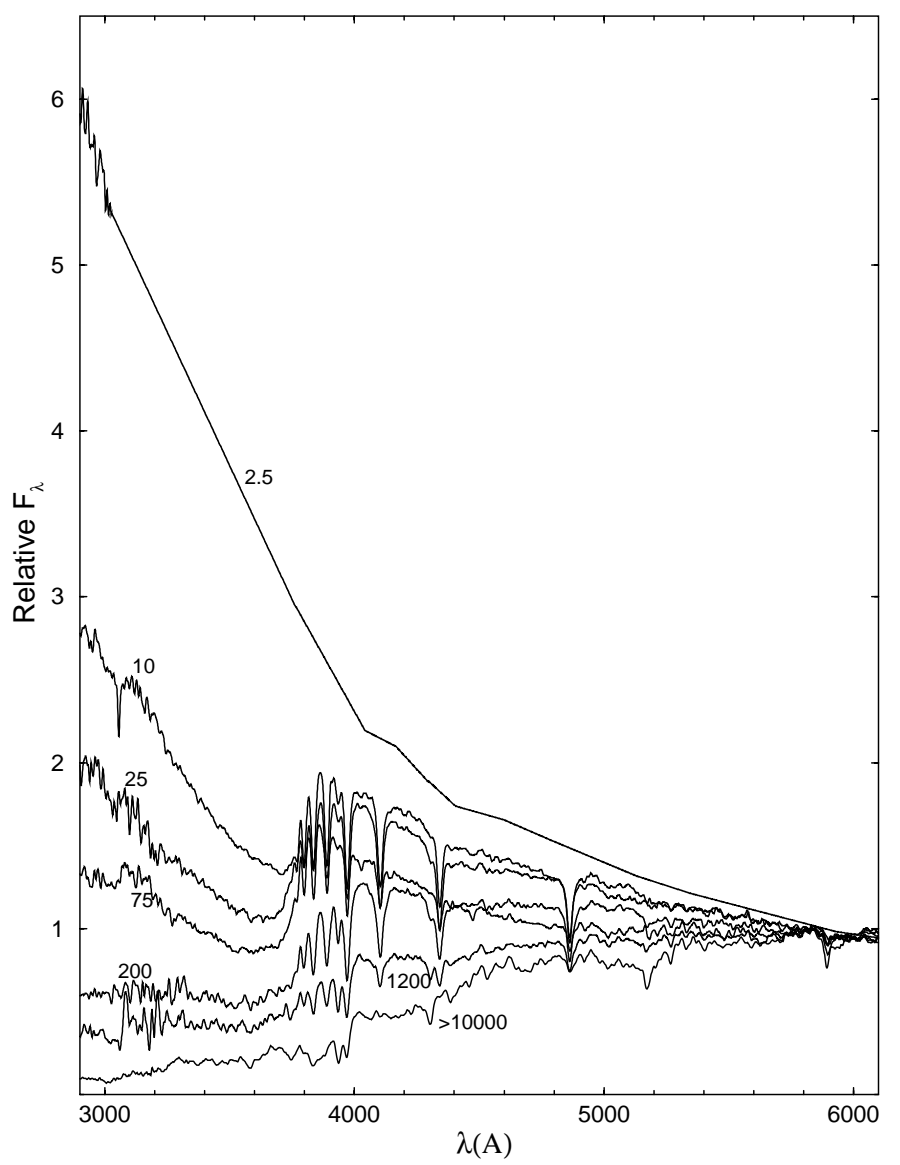

Fig. 7. Spectra templates for the base in the range $\lambda \lambda 2900-6100 \AA$, normalized at $\lambda 5870 \AA$. The spectra are labelled by their respective age (in $10^{6}$ years units).

Initially we use a $10 \%$ step for testing the flux contribution at $5870 \AA$, which means that to each base element will be attributed a multiple of $10 \%$ flux contribution to the total flux, generating about 8000 combinations for each assumed $E(B-V)_{\mathrm{i}}$. Reddenings are tested in the range $0.00 \leq E(B-V)_{\mathrm{i}} \leq$ 0.50 with a step of 0.01 . Thus, in total, 400000 combinations are tested for each running. A second phase consists of exploring at a 5\% step the more restricted space of combinations centred on the family of solutions found during the first phase. This refinement is made by means of successively restrictions of the allowed windows. Next, we calculate the solution with finer steps of $2 \%$ and finally $1 \%$. In this final phase, about $10^{11}$ combinations are generated and the number of acceptable solutions amounts to less than $10^{4}$. We tested the Galactic (Seaton 1979), LMC (Fitzpatrick 1986) and SMC (Prévot et al. 1984) reddening laws for each synthesis.

In principle, a spectral analysis based on eleven features should be sufficient to avoid any non-uniqueness of the solutions as a consequence of the age-metallicity degeneracy. In fact, since our base can be considered complete in terms of age, any discrepancy between galaxy and template metallicity will be reflected and accumulated in the residuals - the difference between observed and synthesized Ws, not affecting, in this way, the age estimates. Consequently, our population age synthesis results can be viewed as acceptable solutions, limited mainly by the intrinsic errors of the observational parameters.

The results of our synthesis are given in Table 5, where we present the percentage contribution of each base element to the flux at $\lambda 5870 \AA$. The values of $E(B-V)_{\mathrm{i}}$ are also given in Table 5. They correspond to the LMC law for the three galaxies.

The component with age $>10 \mathrm{Gyr}$ (hereafter old) dominates the $15870 \AA$ flux in all galaxies. For NGC 1023 and NGC 3115 it contributes, at the nucleus, with about $96-97 \%$ of the flux at $\lambda 5870 \AA$, decreasing outwards to about $82-86 \%$ at 300-400 pc. For NGC 4203, this population shows a different behaviour: increasing contribution from the nucleus $(\approx 90 \%)$ up to $200 \mathrm{pc}(\approx 95 \%)$ and subsequently a decrease; such 


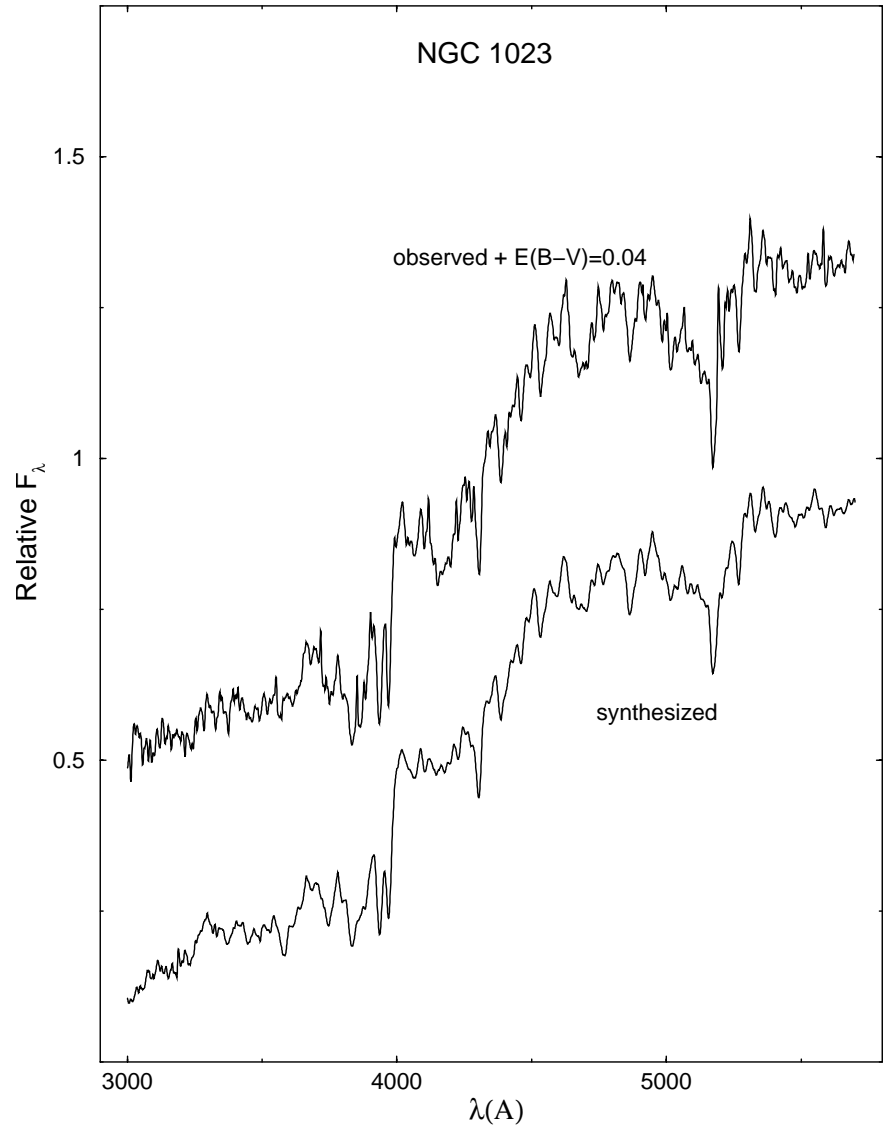

Fig. 8. Observed and synthesized nuclear spectra for NGC 1023, normalized at $\lambda 5870 \AA$. The first one was dereddened with a LMC law and has been shifted by an arbitrary constant, for clarity purposes.

a behaviour could be the result of more recent star-formation in the nucleus or dilution by other continuum sources (e.g. featureless continuum).

The 1.2 Gyr component (hereafter intermediate-age) has a non-negligible contribution in NGC 1023 only from $200 \mathrm{pc}$ outwards (3-5\% of the flux). For NGC 3115 and NGC 4203 this component shows a significant contribution only at $200 \mathrm{pc}$ and $100 \mathrm{pc}$, respectively.

In NGC 1023 the components younger than the intermediate-age population agree, in general, with the contribution of the latter population. For NGC 3115 and NGC 4203 they contribute more significantly at $300 \mathrm{pc}$ in the first case, and at the nucleus, in the second case.

We do not observe a significant gradient of the internal reddening $E(B-V)_{\mathrm{i}}$ for the three galaxies.

In Figs. 8 to 10 we compare the dereddened nuclear observed spectra of the galaxies with the respective synthesized ones. The synthesized spectra were constructed using the star cluster templates (Bonatto et al. 1995) combined according to the proportions given by the synthesis. For NGC 4203 the existence of activity in the nuclear region turns the first two observed spectra into spectra quite different of the synthesized ones, since the base elements have been constructed from star clusters with no emission lines in this spectral region; the synthesis constraints, in this case, are mainly based on the spectral continuum points.

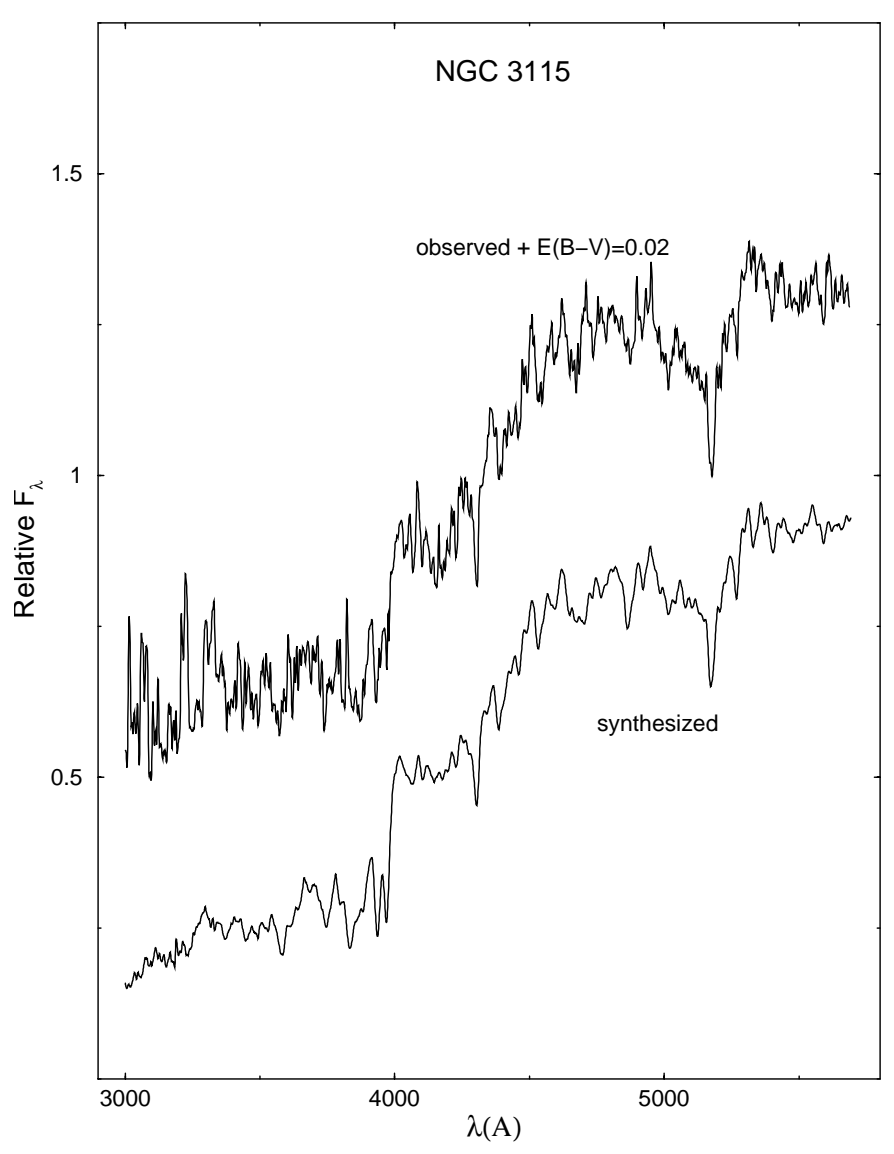

Fig. 9. Same as Fig. 8 for NGC 3115 .

As can be seen in Table 3, most of the absorption features are well reproduced by the synthesis algorithm. For the $\mathrm{CN}$-band feature the difference between the measured Ws and those obtained by the synthesis are more prominent $(\approx 40-60 \%)$, probably due to the strong dependence on metallicity of this feature in the old population (Bica \& Alloin 1986a). In general, the synthesized Ws are smaller than the observed ones, suggesting that the studied galaxies have higher metallicity than the templates.

\subsection{Synthesis results in terms of mass fractions}

The synthesis results (Table 5) refer to flux fractions; in order to discuss star-formation histories it is useful to convert these values into mass fractions. We obtain the corresponding mass fractions by means of mass-to-light ratios $M / L_{\lambda 5870}$ computed from a stellar evolution model of star clusters (Bica et al. 1988). The results are shown in Table 6 . In terms of mass fractions the old stellar population largely dominates $(\approx 98-99 \%)$ the bulge of the three galaxies, demonstrating that most of the gas was converted in stars in the first generation. The intermediate-age population agrees with the distribution derived in the flux fraction analysis: increasing from $200 \mathrm{pc}$ outwards in NGC 1023, with discontinuities in NGC 3115 and NGC 4203 at $200 \mathrm{pc}$ and $100 \mathrm{pc}$, respectively, indicating the possible existence of circumnuclear bursts. The mass fraction stored in young components is negligible for the three galaxies. 
Table 6. Synthesis results in terms of mass fractions.

\begin{tabular}{|c|c|c|c|c|c|c|c|c|}
\hline Element/ag & (10 $0^{6}$ years): & 2.5 & 10 & 25 & 75 & 200 & 1200 & $>10000$ \\
\hline Galaxy & Extraction & \multicolumn{7}{|c|}{ Mass fraction $(\%)$} \\
\hline \multirow{5}{*}{ NGC 1023} & nucleus & $<0.01$ & $<0.01$ & $<0.01$ & $0.01 \pm 0.02$ & $0.02 \pm 0.03$ & $0.23 \pm 0.28$ & $99.73 \pm 0.28$ \\
\hline & $100 \mathrm{pc}$ & $<0.01$ & $<0.01$ & $0.01 \pm 0.01$ & $0.02 \pm 0.02$ & $0.02 \pm 0.03$ & $0.19 \pm 0.25$ & $99.75 \pm 0.25$ \\
\hline & $200 \mathrm{pc}$ & $0.01 \pm 0.01$ & $<0.01$ & $0.03 \pm 0.02$ & $0.07 \pm 0.07$ & $0.05 \pm 0.06$ & $1.78 \pm 0.37$ & $98.06 \pm 0.38$ \\
\hline & $300 \mathrm{pc}$ & $0.01 \pm 0.01$ & $<0.01$ & $0.03 \pm 0.02$ & $0.13 \pm 0.10$ & $0.12 \pm 0.11$ & $0.91 \pm 0.80$ & $98.79 \pm 0.88$ \\
\hline & $400 \mathrm{pc}$ & $0.01 \pm 0.01$ & $<0.01$ & $0.03 \pm 0.02$ & $0.13 \pm 0.10$ & $0.17 \pm 0.14$ & $1.20 \pm 0.97$ & $98.46 \pm 0.92$ \\
\hline \multirow{4}{*}{ NGC 3115} & nucleus & $<0.01$ & $<0.01$ & $<0.01$ & $0.01 \pm 0.02$ & $0.01 \pm 0.02$ & $0.12 \pm 0.20$ & $99.85 \pm 0.20$ \\
\hline & $100 \mathrm{pc}$ & $0.01 \pm 0.01$ & $<0.01$ & $<0.01$ & $0.01 \pm 0.02$ & $<0.01$ & $0.11 \pm 0.19$ & $99.86 \pm 0.19$ \\
\hline & $200 \mathrm{pc}$ & $0.02 \pm 0.00$ & $<0.01$ & $<0.01$ & $0.03 \pm 0.04$ & $0.03 \pm 0.04$ & $1.96 \pm 0.38$ & $97.95 \pm 0.38$ \\
\hline & $300 \mathrm{pc}$ & $<0.01$ & $0.01 \pm 0.01$ & $0.04 \pm 0.01$ & $0.05 \pm 0.06$ & $0.01 \pm 0.02$ & $0.19 \pm 0.25$ & $99.70 \pm 0.26$ \\
\hline \multirow{4}{*}{ NGC 4203} & nucleus & $0.02 \pm 0.00$ & $<0.01$ & $<0.01$ & $0.01 \pm 0.02$ & $0.03 \pm 0.04$ & $0.49 \pm 0.46$ & $99.44 \pm 0.46$ \\
\hline & $100 \mathrm{pc}$ & $<0.01$ & $<0.01$ & $<0.01$ & $0.02 \pm 0.03$ & $0.04 \pm 0.05$ & $2.05 \pm 0.41$ & $97.88 \pm 0.41$ \\
\hline & $200 \mathrm{pc}$ & $<0.01$ & $<0.01$ & $<0.01$ & $0.01 \pm 0.02$ & $<0.01$ & $0.07 \pm 0.12$ & $99.90 \pm 0.12$ \\
\hline & $300 \mathrm{pc}$ & $<0.01$ & $<0.01$ & $<0.01$ & $0.02 \pm 0.03$ & $0.05 \pm 0.05$ & $0.70 \pm 0.66$ & $99.22 \pm 0.66$ \\
\hline
\end{tabular}

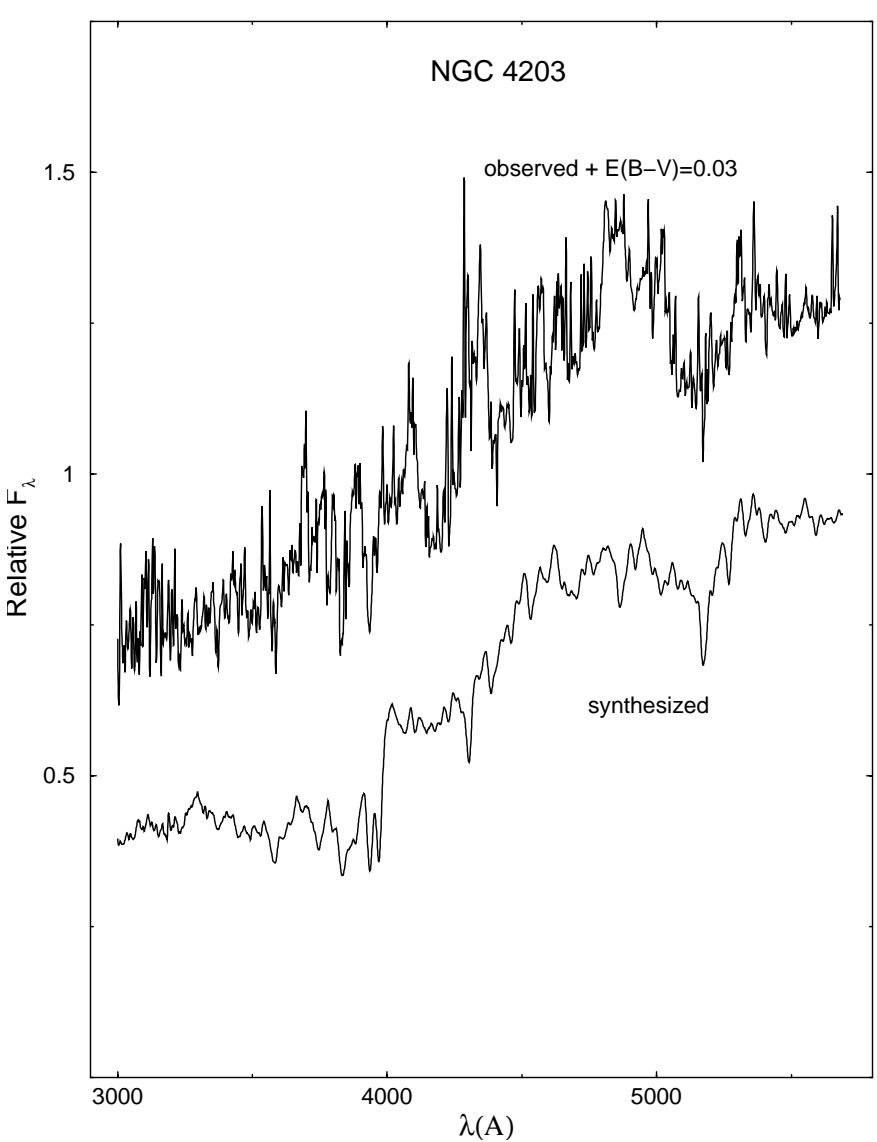

Fig. 10. Same as Fig. 8 for NGC 4203.

\section{Conclusions}

In this work we study the stellar population content in the central parts of three nearby lenticular galaxies using STIS spectra in $\lambda \lambda 2900-5700 \AA$ range obtained by the HST. The analysis was based on a population synthesis method which interprets the variety of populations within the observed regions in terms of age. From the method it is also possible to derive the internal reddening and to infer the associated extinction law.

We presented flux and mass fractions of the different age components derived by the synthesis, inferring also the starformation histories involved. For the three galaxies studied we found that: $i$ ) the old population dominates, both in $\lambda 5870 \AA$ flux fraction and mass fraction; ii) the LMC reddening law is suitable to describe internal extinction; iii) no reddening gradient was found; $i v$ ) there are possible faint CNBs associated to the intermediate-age population in NGC 3115 (at $200 \mathrm{pc}$ from the nucleus) and in NGC 4203 (at 100 pc); $v$ ) the correlation between CNBs and lenticular barred galaxies was not confirmed in our restricted sample.

\section{References}

Arimoto, N., \& Yoshii, Y. 1986, A\&A, 164, 260

Arp, H. C. 1966, ApJS, 14, 1

Athanassoula, E. 1992a, MNRAS, 259, 328

Athanassoula, E. 1992b, MNRAS, 259, 345

Bica, E. 1988, A\&A, 195, 76

Bica, E., \& Alloin, D. 1986a, A\&A, 162, 21

Bica, E., \& Alloin, D. 1986b, A\&AS, 66, 171

Bica, E., Alloin, D., \& Schmitt, H. 1994, A\&A, 283, 805

Bica, E., Arimoto, N., \& Alloin, D. 1988, A\&A, 202, 8

Boissier, S., \& Prantzos, N. 1999, MNRAS, 307, 857

Bonatto, C., Bica, E., \& Alloin, D. 1995, A\&AS, 112, 71

Bonatto, C., Pastoriza, M. G., Alloin, D., \& Bica, E. 1998, A\&A, 334, 439

Bower, G. A., Green, R. F., Bender, R., et al. 2001, ApJ, 550, 75

Capaccioli, M., Held, E. V., \& Nieto, J. L. 1987, AJ, 94, 1519

Capaccioli, M., Lorenz, H., \& Afanasjev, V. L. 1986, A\&A, 169, 54

Charlot, S., \& Bruzual, A. 1991, ApJ, 367, 126 
Clarke, C. 1989, MNRAS, 238, 283

Elmegreen, B. G. 1996, in Cold dust and galaxy morphology, ed. D. Block (Dordrecht: Kluwer)

Fitzpatrick, E. L. 1986, AJ, 92, 1068

Guiderdoni, B., \& Rocca-Volmerange, B. 1987, A\&A, 186, 1

Ho, L. C., Filippenko, A. V., Sargent, W. L. W., \& Peng, C. Y. 1997, ApJS, 112, 391

Illingworth, G., \& Schechter, P. L. 1982, ApJ, 256, 481

Kinney, A. L., Bohlin, R. C., Calzetti, D., Panagia, N., \& Wyse, R. F. G. 1993, ApJS, 86, 5

Kormendy, J. 2001, RMxAC, 10, 69

Lacey, C., \& Fall, M. 1985, ApJ, 290, 154

Larson, R. B., Tinsley, B. M., \& Caldwell, C. M. 1980, ApJ, 237, 692

Martin, P. 1995, AJ, 109, 2428

Molla, M., Ferrini, F., \& Diaz, A. 1997, ApJ, 457, 519

Pastoriza, M. G. 1975, Ap\&SS, 33, 173

Pease, F. 1917, ApJ, 46, 24

Persson, S. E., Frogel, J. A., \& Aaronson, M. 1979, ApJS, 39, 61

Pickles, A. J. 1985, ApJ, 296, 340

Piner, B. G., Stone, J. M., \& Teuben, P. J. 1995, ApJ, 449, 508

Pogge, R. W., \& Eskridge, P. B. 1987. AJ, 93, 291
Prévot, M. L., Lequeux, J., Maurice, E., Prévot, L., \& Rocca-Volmerange, B. 1984, A\&A, 132, 389

Sancisi, R., van Woerden, H., Davies, R. D., \& Hart, L. 1984, MNRAS, 210, 514

Sarzi, M., Rix, H.-W., Shields, J. C., et al. 2001, ApJ, 550, 65

Schmitt, H. R., Bica, E., \& Pastoriza, M. G. 1996, MNRAS, 278, 965

Seaton, M. J. 1979, MNRAS, 187, 73p

Sil'chenko, O. K. 1999, AJ, 117, 2725

Silk, J., \& Norman, C. 1979, ApJ, 234, 86

Silva, D. R., Boroson, T. A., Thompson, I. B., \& Jedrzejewski, R. I. 1989, AJ, 98, 131

Tinsley, B. M. 1980, ApJ, 241, 41

van den Bergh, S. 1975, ARA\&A, 13, 217

van Driel, W., van Woerden, H., Gallagher, J. S., \& Schwarz, U. J. 1988, A\&A, 191, 201

van Woerden, H., van Driel, W., \& Schwarz, U. J. 1983, in Internal kinematics and dynamics of galaxies, IAU Symp. 100, ed. E. Athanassoula (Dordrecht: Reidel)

de Vaucouleurs, G., \& de Vaucouleurs, A. 1964, Reference catalogue of bright galaxies (Austin: University of Texas Press)

Williams, T. B. 1976, ApJ, 209, 716 